\title{
BMJ Open The job content questionnaire in various occupational contexts: applying a latent class model
}

\author{
Kionna Oliveira Bernardes Santos, ${ }^{1}$ Tânia Maria de Araújo, ${ }^{2}$ \\ Fernando Martins Carvalho, ${ }^{3}$ Robert Karasek ${ }^{4,5}$
}

To cite: Santos KOB, Araújo TMde, Carvalho FM, et al. The job content questionnaire in various occupational contexts: applying a latent class model. BMJ Open 2017;7:e013596. doi:10.1136/ bmjopen-2016-013596

- Prepublication history and additional material are available. To view please visit the journal (http://dx.doi.org/ 10.1136/ bmjopen-2016-013596).

Received 30 July 2016 Revised 23 February 2017 Accepted 29 March 2017

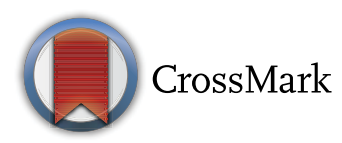

${ }^{1}$ Department of Physiotherapy, Federal University of Bahia (Universidade Federal da Bahia: UFBA), Salvador, Bahia, Brazil ${ }^{2}$ Department of Health, State University of Feira de Santana (Universidade Estadual de Feira de Santana: UEFS), Feira de

Santana, Bahia, Brazil

${ }^{3}$ Department of Preventive and Social Medicine, Universidade Federal da Bahia: UFBA,

Salvador, Bahia, Brazil

${ }^{4}$ Professor Emeritus, Department of Work Environment, University of Massachusetts, Lowell, USA

${ }^{5}$ Professor Emeritus, Institute for Psychology, Copenhagen University, Denmark

Correspondence to Dr Kionna Oliveira Bernardes Santos;

kionna.bernardes@gmail.com

\section{ABSTRACT}

Objective To evaluate Job Content Questionnaire(JCQ) performance using the latent class model.

Methods We analysed cross-sectional studies conducted in Brazil and examined three occupational categories: petroleum industry workers $(n=489)$, teachers $(n=4392)$ and primary healthcare workers (3078)and 1552 urban workers from a representative sample of the city of Feira de Santana in Bahia, Brazil. An appropriate number of latent classes was extracted and described each occupational category using latent class analysis, a multivariate method that evaluates constructs and takes into account the latent characteristics underlying the structure of measurement scales. The conditional probabilities of workers belonging to each class were then analysed graphically.

Results Initially, the latent class analysis extracted four classes corresponding to the four job types (active, passive, low strain and high strain) proposed by the Job-Strain model (JSM) and operationalised by the JCQ. However, after taking into consideration the adequacy criteria to evaluate the number of extracted classes, three classes (active, low strain and high strain) were extracted from the studies of urban workers and teachers and four classes (active, passive, low strain and high strain) from the study of primary healthcare and petroleum industry workers.

Conclusion The four job types proposed by the JSM were identified among primary healthcare and petroleum industry workers - groups with relatively high levels of skill discretion and decision authority. Three job types were identified for teachers and urban workers; however, passive job situations were not found within these groups. The latent class analysis enabled us to describe the conditional standard responses of the job types proposed by the model, particularly in relation to active jobs and high and low strain situations.

\section{INTRODUCTION}

Since its development more than 30 years ago, the Job-Strain model (JSM) has been widely used to explain the psychosocial aspects of work. The model provides the theoretical framework that underpins the Job Content Questionnaire (JCQ), an instrument to measure the psychosocial aspects of work, which presupposes four types of job

\section{Study strengths and limitations}

Previous studies have confirmed the Job Content Questionnaire (JCQ) dimensional structure using factor analysis, but none have used latent class analysis.

- The study confirmed the adequacy of the JCQ's theoretical framework across different groups of workers. The latent class analysis identified response profiles equivalent to those defined by the JCQ quadrants.

- This statistical technique enabled the identification of those items from each JCQ scale or subscale most relevant to the profiles of the occupational groups investigated.

experience. Job types derive from a combination of 'high' and 'low' levels of psychological job demands and worker decision latitude: high strain jobs (characterised by high psychological demands and low decision latitude), active jobs (high psychological demands and high decision latitude), passive jobs (low psychological demands and low decision latitude) and low strain jobs (low psychological demands and high decision latitude). ${ }^{1}$

The scientific literature contains a fertile discussion about JSM adequacy and inconsistencies. However, meta-analyses indicate that JSM dimensions (demand and control) are good predictors of important worker health outcomes. $^{23}$

Subjective concepts such as job control take on different meanings depending on cultural origins. ${ }^{4}$ The JSM has been evaluated in different cultures and job contexts. However, most of the results available in the literature come from Western job contexts, raising concerns about the model's validity in different cultures. ${ }^{5}$

The original $\mathrm{D} / \mathrm{C}$ model has deep roots in organisational sociology, focusing on the organisation's work output goals (Control) and its division of labour and skill allocation 
structure (Demand). JCQ individual-level measurement also allowed its use in organisational psychology and social epidemiology research. JCQ questions showed good validity and reliability in formal and informal jobs. ${ }^{6}$

Overall, the JCQ dimensional structure is considered adequate. $^{7-11}$ However, certain studies have produced inconsistent data when evaluating JCQ subscales and have indicated the need for more detailed investigations into the interactions between its main dimensions and its subscales. ${ }^{12-18}$

The various JCQ versions and methodologies used to evaluate JSM constructs present challenges of a psychometric nature. In this context, the construct is taken as a hypothesis or an explanation for a specific performance in relation to the results achieved. The more complex the theoretical model, the more difficult the interpretation of its criteria for predicting results. ${ }^{19}$

There are, therefore, gaps in the literature regarding the JSM's theoretical implications, the JCQ subscales and the structure of answers in terms of their adequacy in measuring occupational stressors. Despite discussions about the limits and progress of using the JSM to evaluate psychosocial work aspects, a mass of scientific literature and empirical material exists, which bases its application on an analysis of work organisation elements and their impacts on worker health. However, studies in several cultures and work contexts have revealed inconsistencies in some JCQ items, suggesting changes to be incorporated into the model's evaluation in order to increase its predictive capacity. ${ }^{6} 1011$ 13-17 20-23

Latent class analysis evaluates constructs (theoretical variables) indirectly, by means of two or more observed variables. Latent class models are applied when both the variables and their indicators are categorical. Moreover, although this is a multivariate method, latent class analysis enables an understanding of complex relationships, which is impossible to achieve by other methods. ${ }^{21}$

This study aimed to evaluate the performance of the $\mathrm{JCQ}$ in a range of occupational contexts using latent class analysis.

\section{METHODS}

The study investigated 9511 Brazilian workers, selected by specific sampling procedures in four cross-sectional design studies, from four different occupational contexts.

Study 1: Industry workers. A census of the permanent workers $(n=489)$ working in the petroleum industry in the State of Bahia, Brazil, in 2008. ${ }^{24}$

Study 2: Teachers. A census of the 4392 teachers in the 365 public schools in the municipal educational system in Salvador, Brazil in 2006. ${ }^{25}$ The municipal educational system covers kindergarten and primary school (covering school years 1-8).

Study 3: A study from 2012 to 2013 involving 3078 primary healthcare workers from five municipalities in the State of Bahia, Brazil (Feira de Santana, Jequié, Santo Antônio de Jesus, Itabuna and a Salvador health district).
A stratified proportional sample took into account individual distribution according to geographical area and occupational group. ${ }^{26}$

Study 4: Urban workers. A random sample of 1552 individuals, representing workers over 15 years of age, stratified according to sub-districts in the urban area of Feira de Santana in 2007. ${ }^{27}$

Female participation varied from $54.7 \%$ in the study of urban workers to $92.0 \%$ in the teacher study. An age range of between 31 and 45 years prevailed in the four studies, varying from $37.6 \%$ of the total urban workers to $53.2 \%$ of the teachers. Educational level differed among the occupational categories: undergraduate level predominated among the industry workers and the teachers $(56.6 \%$ and $83.7 \%$, respectively), whereas high school level prevailed $(53.0 \%)$ among the health workers.

The JCQ was used to evaluate psychosocial work aspects in the four studies. This instrument poses questions in LikertScale format, varying from 1 ('strongly disagree') to 4 ('strongly agree'). In all the studies, the decision latitude (skill discretion + decision authority) and psychological demands scales were used.

Latent class analysis was performed separately on each data set. The classes were first described and the conditional probabilities of the items belonging to each class were then analysed. Adjustments to the questionnaire response items were made in order to facilitate analysis. Certain items were, therefore, inverted: Q2 'repetitive' and Q9 'little decision freedom', in the decision latitude scale; and Q10 'no excessive work' and Q13 'enough time', in the psychological demands scale (see online Supplementary appendix 1). The answers to the questions were dichotomised as follows: 'strongly disagree' and 'disagree' $=0$; 'strongly agree' and 'agree' $=1$.

The Vuong-Lo-Mendell-Rubin, Lo-Mendell-Rubin likelihood ratio test (LRT) adjusted and maximum likelihood parametric bootstrap tests were used to evaluate the adequacy of the number of extracted classes. A criterion for the three tests' agreement was adopted in order to accept the number of extracted classes. For the purposes of these analyses, we adopted a $\mathrm{p}$ value $<0.05$.

Entropy measures were analysed in order to evaluate the goodness of class separation. Values above 0.80 were considered good. ${ }^{28}$

Conditional probabilities were presented graphically. The questionnaire items were plotted on the X-axis, while the Y-axis represented the probability of answering 'yes' to a specific item and thus belonging to a specific class. Answers with a probability above 0.5 were considered to represent an interpretation criterion. This analysis used MPLUS software, version $7{ }^{29}$

Prior to their initiation, the four studies were approved by their respective Ethics Committees. This study was approved by the Ethics Committee-CAAE under no: 18723813.9.0000.5030. 
Table 1 Populations studied according to the number of classes in the Job-Strain model and the entropy and adjustment criteria for the number of extracted classes

\begin{tabular}{|c|c|c|c|c|c|c|c|c|}
\hline & \multicolumn{2}{|c|}{$\begin{array}{l}\text { Industry workers } \\
(\mathrm{n}=489)\end{array}$} & \multicolumn{2}{|c|}{$\begin{array}{l}\text { Teachers } \\
(n=4.392)\end{array}$} & \multicolumn{2}{|c|}{$\begin{array}{l}\text { Health workers } \\
(\mathrm{n}=3.078)\end{array}$} & \multicolumn{2}{|c|}{$\begin{array}{l}\text { Urban workers } \\
(n=1.552)\end{array}$} \\
\hline & $\mathbf{n}$ & $(\%)$ & $\mathbf{n}$ & $(\%)$ & $\mathbf{n}$ & $(\%)$ & $\mathbf{n}$ & (\%) \\
\hline High strain & 68 & 13.7 & 747 & 18.5 & 244 & 9.1 & 455 & 30.2 \\
\hline Active job & 151 & 30.9 & 1237 & 29.0 & 889 & 28.8 & 472 & 30.7 \\
\hline Passive job & 75 & 15.5 & & & 811 & 26.9 & & \\
\hline Low strain & 195 & 39.9 & 2408 & 52.5 & 1134 & 35.2 & 625 & 39.1 \\
\hline Entropy & 0.77 & & 0.61 & & 0.62 & & 0.60 & \\
\hline VLMR test* & 0.01 & & 0.01 & & 0.04 & & 0.01 & \\
\hline LMR-LRT test $†$ & 0.01 & & 0.01 & & 0.04 & & 0.01 & \\
\hline PB test $\neq$ & 0.01 & & 0.01 & & 0.04 & & 0.01 & \\
\hline
\end{tabular}

*VLMR, Vuong-Lo-Mendell-Rubin test $-\mathrm{p}$ value.

†LMR-LRT, Lo Mendell Rubin test, adjusted (LRT test) - $p$ value.

$\ddagger$ PB, Maximum Likelihood Parametric Bootstrapped test $-p$ value.

\section{RESULTS}

The latent class analysis initially extracted four classes, in line with the four job types proposed by the JSM (high strain, low strain, active and passive). However, after considering the adequacy criteria for evaluating the number of extracted classes, the studies differed: three classes were extracted from studies 2 and 4 and four classes from studies 1 and 3 .

The entropy measure had a higher score in relation to the study 1 classes $(0.77)$, while its lowest value was found in study $4(0.60)$. All other tests to evaluate the number of classes in the studies demonstrated very low $p$ values (table 1).

The latent class analysis extracted four classes from study 1 (workers in the petroleum industry). One class classified $30.9 \%$ of the workers as working in an active job. This class included workers with a high probability of agreeing to the items related to skill discretion and decision authority (Q1-Q9) that form decision latitude and a higher probability of providing positive responses to the items related to psychological demands (Q10-Q14) (figure 1).

The analysis also revealed that $39.9 \%$ of petroleum industry workers were in a low strain job. Workers in this class had a higher probability of agreeing to items related to the decision latitude dimension and a lower probability of giving positive answers to items related to psychological demand. The third class identified in study 1 assessed $15.5 \%$ of the workers as being in a passive job. The fourth class described a situation analogous to a high strain job (13.7\%). Although the skills discretion items had a high probability of response (Q2-Q4), low control was marked by decision authority items. Items relating to psychological demands demonstrated a high probability of response in this class.

Workers in the high strain quadrant presented high positive probability in the issues Q12 'work fast' and Q11 'work hard', denoting high psychological job demands.
Study 2 (teachers) presented the highest proportion of workers with the probability of belonging to a low strain job $(52.5 \%)$, followed by an active job (29.0\%). The workers in the remaining class $(18.5 \%)$ presented the lowest probability of positive responses to the items on the decision latitude subscales, particularly to decision authority (Q7-Q9) and high levels of agreement with the items related to psychological demands. For this reason, teachers in this class were classified into the high strain job type (figure 2).

For the teachers, there was a positive probability for items relating to skill discretion, except in the high-demand group, in which we observed a low probability of certain skill discretion items (Q5 'variety' and Q6 'develop own abilities')

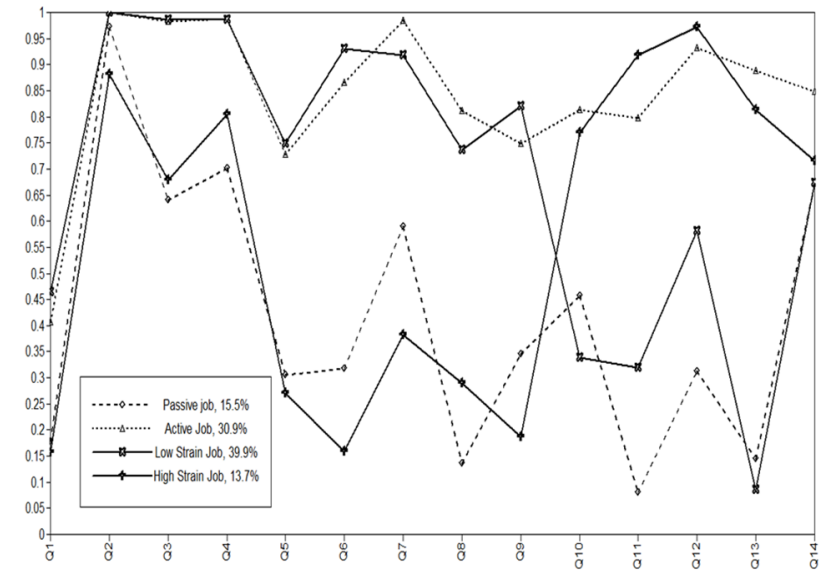

Figure 1 Q1 - 'learn new things', Q2 - 'repetitive', * Q3'requires creativity', Q4 - 'high skill level', Q5- 'variety', Q6-'develop own abilities', Q7 - 'lot of say', Q8- 'allow own decisions', Q9- 'little decision freedom', , Q10- 'no excessive work', ${ }^{\star}$ Q11 - 'work hard', Q12 - 'work fast', Q13-'enough time *' and Q14- 'conflict demands'. *Items inverted for calculation purposes. 


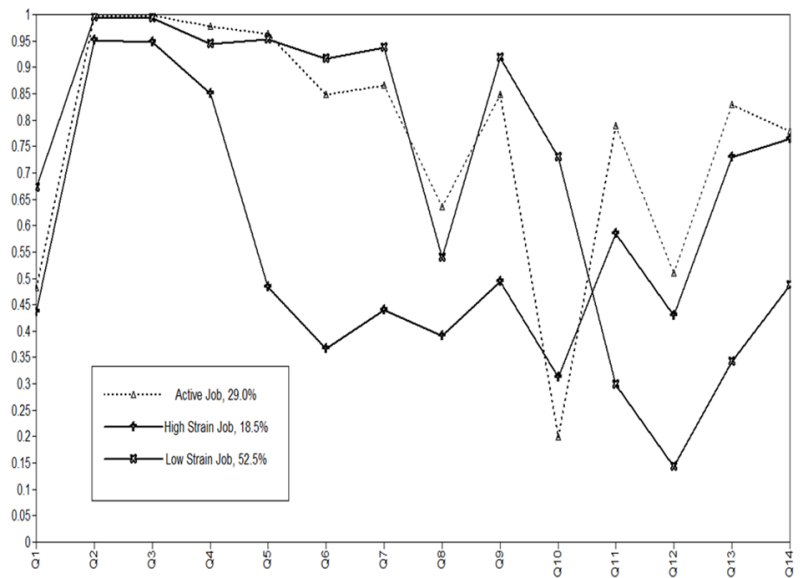

Figure 2 Q1 - 'learn new things', Q2 - 'repetitive', , Q3'requires creativity', Q4 - 'high skill level', Q5- 'variety', Q6- 'develop own abilities', Q7 - 'lot of say', Q8- 'allow own decisions', Q9- 'little decision freedom', * Q10- 'no excessive work', $\mathrm{Q} 11$ - ‘work hard', Q12 - 'work fast', Q13-'enough time *' and Q14- 'conflict demands'. *Items inverted for calculation purposes.

In study 3 (primary healthcare workers), conditional probabilities allocated the greatest proportion $(35.2 \%)$ of individuals into a class defined as a low strain job. This class included workers with a high probability of agreement about items related to job control, but a low probability of providing positive answers to the items related to psychological demands. The passive job quadrant encompassed $28.8 \%$ of these workers, who presented a low/moderate probability of positive responses to items from both the decision latitude and psychological demands scales. In this class, the performance of item Q13 'enough time' (inverted) was noteworthy, indicating that the time for workers to carry out their tasks was adequate (figure 3).

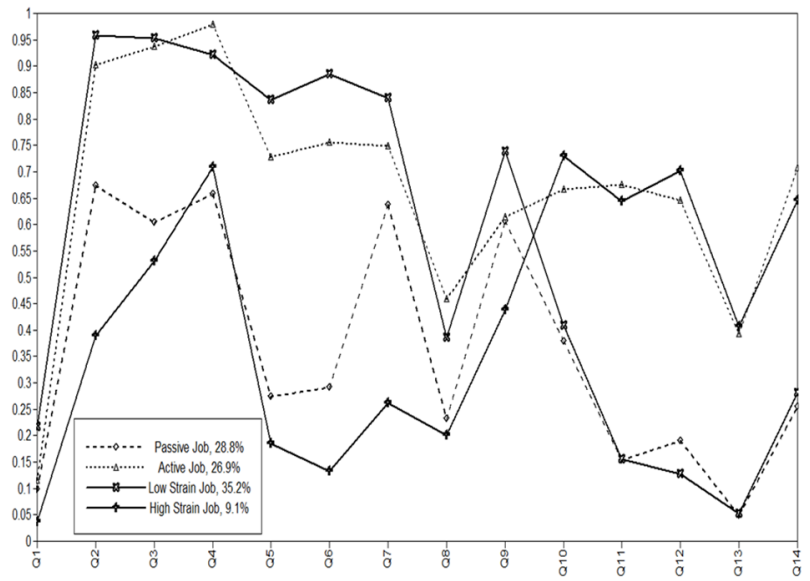

Figure 3 Q1 - 'learn new things', Q2 - 'repetitive', , Q3'requires creativity', Q4- ‘high skill level', Q5- 'variety', Q6- 'develop own abilities', Q7 - 'lot of say', Q8 - 'allow own decisions', Q9- 'little decision freedom', * Q10- 'no excessive work', $Q 11$ - 'work hard', Q12 - 'work fast', Q13- 'enough time *' and Q14- 'conflict demands'. *Items inverted for calculation purposes.



Figure 4 Q1-'learn new things', Q2 - 'repetitive', , Q3'requires creativity', Q4- 'high skill level', Q5- 'variety', Q6- 'develop own abilities', Q7 - 'lot of say', Q8 - 'allow own decisions', Q9 - 'little decision freedom', ' Q10-'no excessive work', $\mathrm{Q} 11$ - 'work hard', Q12 - 'work fast', Q13- 'enough time *' and Q14- 'conflict demands'. 'Items inverted for calculation purposes.

One class presented characteristics analogous to the situation of a high strain job $(9.1 \%)$. Higher probabilities were found regarding psychological demands in items Q10 'no excessive work' (inverted) and Q12 'work fast'. The items in the decision authority subscale provided lower positive probabilities, yielding low probabilities for the control scale, highlighting the high demands in the high-demand group and the low probability for items related to decision authority.

Study 4 presented only three classes (figure 4). Despite the high/moderate probability of positive responses to the skill discretion items, three types of work were distinguished: low strain job $(39.1 \%)$, active job $(30.7 \%)$ and high strain job $(30.2 \%)$. Among urban workers, the psychological demand items presented conditional probability answers slightly above 0.5 . Such probability was taken as a criterion for the control scale (Skill Discretion) items when classifying a high strain job. Item Q10 'no excessive work' (inverted) demonstrated a significant contribution to the level of psychological demands.

\section{DISCUSSION}

Latent class analysis allowed us to extract a varied number of classes from the four studies. Using this technique enabled an evaluation of the patterns of responses to categorical variables and a description of the clustering of observations. ${ }^{29}$ Furthermore, this technique identified which items from each scale or subscale were more relevant to the profiles of each occupational group. This is an important and applicable feature of this latent class analysis: beyond the JCQ scales evaluation, it makes it possible to determine which specific aspects could be remodelled in work processes and organisation. This information may be useful in planning interventions in the work environment. 
The extracted classes revealed the varied composition of psychosocial aspects, according to occupational categories. The latent class model was based on conditional patterns of positive responses associated with the extracted classes, leading to the discrimination of subgroups, independent of methodological criteria or the JCQ recommended calculations. Moreover, the results enabled an item-by-item evaluation.

The JSM has confirmed its theoretical assumption, which combines the control and psychological job demand dimensions in the studies we analysed. However, the whole JCQ quadrant structure, as proposed by the JSM, was only identified in two of the four studies.

Most of the studies we analysed had large sample sizes and were relatively homogeneous, enabling us to meet the criteria for the application of an analytical technique and an evaluation of the main JSM dimensions. Population characteristics and the method chosen to evaluate psychosocial work aspects may affect model performance. $^{2030}$

Isolating occupational categories would allow for a more detailed analysis of the organisational characteristics of a specific activity, increasing the JSM's predictive capacity ${ }^{31}$ However, criticisms still remain regarding the low sensitivity of JCQ items in detecting psychosocial work aspects, even in homogeneous samples. ${ }^{32}$

Criticisms also persist regarding the main dimensions that underlie the JSM, particularly regarding the assumption of independence between its scales, translated into an orthogonal relationship in the analysis. This argument takes into consideration the interdependence that exists between these dimensions. Investigations must, therefore, take into account these possible connections and their effects on the job types proposed by the model. ${ }^{12} 33$

To the best of our knowledge, no study has evaluated the JSM assumptions using the latent class approach. According to this analytical technique, data are generated by a hierarchical process, so that each individual becomes a member of a determined class (latent factor). The job types proposed by the Demand-Control Model were found in the studies we analysed, with a predominance of low strain and active jobs in the occupational groups. Low strain jobs represented the greatest proportion of conditional probabilities in the four studies. Despite the different work contexts, the occupational categories presented a reasonable degree of freedom to act. Jobs that involve autonomy and professional recognition (physicians, nurses, teachers, engineers, lawyers, etc.) have been classified within this typology. ${ }^{34}$ Professionals in active jobs were also prominent in the three occupational groups we studied. This result is compatible with the JSM forecasts.

Active jobs, characterised by high psychological demands and high control over one's own work, provide motivation and support the learning of new skills. ${ }^{34}$ However, the positive effect of control in a situation of occupational stress has been questioned. ${ }^{35}$ The authority decision items subscale presented high agreement levels in situations of high control. Some items on the skill discretion subscale demonstrated the high probability of response in situations of high psychological demand.

Low strain jobs were most frequently found in the teacher study $(52.5 \%)$, followed by the industry worker study $(39.9 \%)$ and the urban worker one $(39.1 \%)$. Item Q2 'repetitive' (inverted) presented low probability in all studies. Analyses of the JCQ dimensional structure demonstrated the difficulty of measuring this item using the skill discretion subscale. ${ }^{13} 1721$

We found that in occupational categories where task responsibility level is high, psychological demands are similarly high. If one is continuously seeking to develop professionally, the high level of control primarily relates to psychological demands. ${ }^{36-38}$ In these situations, control cannot prevent the negative effects of high demands. ${ }^{9}{ }^{36}$ In other words, when job demands are relatively high, exceeding worker control, a condition similar to learned helplessness may occur. Such a sense (of the inability to influence work demands) makes workers more vulnerable and can reduce the beneficial effects associated with high levels of control. ${ }^{39}$

The health and education fields are not developing well in Brazil. The target population is in poor health; resources for professional practice are scarce or inadequate and work management models are authoritative, highly hierarchical and ineffective in coordinating the activities required to respond to population demand. Demands for health and education services are increasingly high and reflect marked inequalities and social exclusion. Public and private sector activities in these fields are unable to produce effective results. For workers in the areas of health and education, this adverse situation generates a sense of ineffectiveness and a lack of recognition of their professional efforts. The active job style and its effects on the health of workers from countries with occupational contexts similar to Brazil deserve further attention, so as to reach a better understanding of the relationships between demand and control in these situations.

The class of workers with a low probability of positive responses to the control and psychological demands subscales suggested passive jobs, as observed among the primary healthcare $(28.8 \%)$ and petroleum industry workers $(15.5 \%)$. This result agrees with the work management models prevalent in Brazil, characterised by authoritative and unequal relationships. Nevertheless, certain skill discretion items presented high probabilities. The two subscales for control increased the complexity of analysis but enabled stronger correlations with those for psychological demands. Moreover, the use of affective judgements when using this scale, as well as the work context, may bias the measures and deviate from the JSM's theoretical principles. ${ }^{36} 38$

High strain job situations were only found in the studies of healthcare and industry workers. Items Q10 'no excessive work' (inverted), Q11 'work hard' and Q12 'work fast' presented high conditional probabilities. For 
workers in this job type, most items from the control scale demonstrated low probability, except for item Q4 'high skill level'.

These results are consistent with the characteristics of jobs in health, since, to a lesser or greater degree, these activities require specialised technical knowledge, regardless of other work factors that may require specific skills.

Different cultures develop different strategies to cope with psychosocial job characteristics. This was not, however, the focus of this study. We evaluated different occupational groups from different economic sectors. This approach can be useful in understanding specific job characteristics and the sociocultural contexts which structure specific occupational stressors and enable different job situations (quadrants) to predominate, as predicted by the demand-control model. This finding supports the idea that tools designed for use in different occupational contexts, such as the JCQ, can also be used to identify specific critical situations.

This study demonstrates that JCQ performance differs across different occupational contexts in Brazil. It is worth noting that unless the entire population is included-and, in particular, if certain occupational groups dominate the sample - then, one would not expect to see the full set of quadrants represented to the same extent. Our data did not allow us to discuss the JCQ performance across different countries and cultures. The Occupational Maps presented by Karasek and Theorell ${ }^{34}$ demonstrate very specific differences that one would expect in samples from different occupational groups.

Here, we are applying a different method than the standard one used by Karasek and colleagues ${ }^{34}$ It is important to recognise that certain adjustments made in order to apply the analytical technique may have interfered in the interpretation of the results. Variable dichotomization and the fact that we did not use the formulas originally proposed for the JSM calculations may have affected our results. Researchers always learn something new when they face the challenge of building new analytical perspectives to broaden the understanding of the aspects studied, by using an entirely different methodological. From our study, we have learned that latent class analysis allowed us to observe the workers' conditional pattern of responses by taking into account combinations of the JSM main dimensions.

\section{CONCLUSION}

This study applied a new statistical method for analysing psychological work aspects, taking into account the latent characteristics underlying the measurement scales' structure. Although the four job types proposed by the JSM were not identified in all the studies, latent class analysis allowed us to describe the conditional standard responses of the job types proposed by the model, particularly regarding active jobs, passive jobs and low strain situations.
The study confirmed the adequacy of the theoretical framework assumed by the JCQ across different worker categories. Latent class analysis identified response profiles equivalent to those defined by the JCQ quadrants.

Contributors We declare that all the authors of this paper fulfil the authorship criteria. KOBS, FMC and TMdA worked on study conception and design, analysis, interpretation, writing and reviewing and approved the final version for publication. RK worked on study interpretation, reviewing and approved the final version for publication.

Competing interests None declared.

Ethics approval Ethical Committee (CAAE 18723813.9.0000.5030).

Provenance and peer review Not commissioned; externally peer reviewed.

Open Access This is an Open Access article distributed in accordance with the Creative Commons Attribution Non Commercial (CC BY-NC 4.0) license, which permits others to distribute, remix, adapt, build upon this work non-commercially, and license their derivative works on different terms, provided the original work is properly cited and the use is non-commercial. See: http://creativecommons.org/ licenses/by-nc/4.0/

(C) Article author(s) (or their employer(s) unless otherwise stated in the text of the article) 2017. All rights reserved. No commercial use is permitted unless otherwise expressly granted.

\section{REFERENCES}

1. Karasek RA. Job demands, job decision latitude, and mental strain: implications for job redesign. Adm Sci Q 1979;24:285-308.

2. Häusser JA, Mojzisch A, Niesel M, et al. Ten years on: a review of recent research on the job Demand-Control (-Support) model and psychological well-being. Work Stress 2010;24:1-35.

3. Luchman JN, González-Morales MG. Demands, control, and support: a meta-analytic review of work characteristics interrelationships. J Occup Health Psychol 2013;18:37-52.

4. Verhoeven C, Maes S, Kraaij V, et al. The job Demand-Control-Social support model and wellness/Health outcomes: a European study. Psychol Health 2003;18:421-40.

5. Ibrahim R, Ohtsuka K. Review of the job Demand-Control and job Demand-Control-Support models: elusive moderating predictor effects and cultural implications. Southeast Asia Psychol J 2012;1:10-21.

6. Araújo TM, Karasek R. Validity and reliability of the job content questionnaire in formal and informal jobs in Brazil. Scand $J$ Work Environ Health 2008;34:52-9.

7. Niedhammer I, Chastang JF, Gendrey L, et al. Propriétés psychométriques de la version française des échellesde la demande psychologique, de la latitude décisionnelle et Du Soutien social Du «Job Content Questionnaire» de Karasek: résultats de l'enquête nationale SUMER. Sante Publique 2006;18:413-27.

8. Eum KD, Li J, Jhun HJ, et al. Psychometric properties of the Korean version of the job content questionnaire: data from health care workers. Int Arch Occup Environ Health 2007;80:497-504.

9. Phakthongsuk P. Construct validity of the Thai version of the job content questionnaire in a large population of heterogeneous occupations. J Med Assoc Thai 2009;92:564-72.

10. Choobineh A, Ghaem H, Ahmedinejad P. Validity and reliability of the Persian (Farsi) version of the Job Content Questionnaire: a study among hospital nurses. East Mediterr Health J 2011;17:335-41.

11. Nehzat F, Huda BZ, Tajuddin SH. Reliability and validity of job content questionnaire for university research laboratory staff in Malaysia. Southeast Asian J Trop Med Public Health 2014;45:481-9.

12. Kain J, Karasek's JS. Job demand-control model: a summary of current issues and recommendations for future research. In: Perrewé PL, Ganster DC, eds. New developments in theoretical and conceptual approaches to job stress. . Bingley, West Yorkshire: Emerald Group Publishing, 1979:2010. 237-68.

13. Hökerberg $\mathrm{YH}$, Aguiar OB, Reichenheim M, et al. Dimensional structure of the demand control support questionnaire: a Brazilian context. Int Arch Occup Environ Health 2010;83:407-16.

14. Griep $\mathrm{RH}$, Rotenberg $\mathrm{L}$, Landsbergis $P$, et al. Combined use of job stress models and self-rated health in nursing. Rev Saude Publica 2011;45:145-52.

15. Mase J, Ota A, Inoue K, et al. Reliability and validity of the Japanese translated version of the Swedish Demand-Control-Support Questionnaire. Ind Health 2012;50:467-75. 
16. Jabali SMT, Ghaffari M, Pournik O, et al. Reliability and validity of Persian version of job content questionnaire in health care workers in Iran. Int J Occup Environ Med 2013;4b:96-101.

17. Chungkham HS, Ingre M, Karasek R, et al. Factor structure and longitudinal measurement invariance of the demand control support model: an evidence from the Swedish longitudinal occupational survey of health (SLOSH). PLoS One 2013;8:e70541-11.

18. Hökerberg $\mathrm{YH}$, Reichenheim ME, Faerstein $\mathrm{E}$, et al. Cross-cultural validity of the demand-control questionnaire: swedish and brazilian workers. Rev Saude Publica 2014;48:486-96.

19. Streiner DL, Norman GR. Health measurement scales: a practical guide to their development and use. 4th edn. Oxford: Oxford University Press, 2008.

20. Phakthongsuk $P$, Apakupakul N. Psychometric properties of the Thai version of the 22-item and 45 -item Karasek job content questionnaire. Int J Occup Med Environ Health 2008;21:331-44.

21. Niedhammer I. Psychometric properties of the French version of the Karasek Job Content Questionnaire: a study of the scales of decision latitude, psychological demands, social support, and physical demands in the GAZEL cohort. Int Arch Occup Environ Health 2002;75:129-44.

22. Sale JE, Kerr MS. The psychometric properties of Karasek's demand and control scales within a single sector: data from a large teaching hospital. Int Arch Occup Environ Health 2002;75:145-52.

23. Choi B, Kawakami N, Chang S, et al. A cross-national study on the multidimensional characteristics of the five-item psychological demands scale of the Job Content Questionnaire. Int J Behav Med 2008:15:120-32.

24. Fonseca ISS, Araújo TM, Santos KOB, et al. Apoio social e satisfação no trabalho em funcionários de Uma empresa de petróleo. Psicol Am Lat 2014;25:43-56.

25. Cardoso JP, Araújo TMde, Carvalho FM, et al. Aspectos psicossociais do trabalho e dor musculoesquelética em professores. Cad Saude Publica 2011;27:1498-506.

26. Santos KOB, Carvalho FM, Araújo TM. Consistencia Interna do SelfReporting Questionnaire-20 em grupos ocupacionais. Rev Saude Publica 2016;50:1-10.
27. Prisco APK, Araújo TM, Almeida MMG, et al. Prevalência De transtornos alimentares em trabalhadores urbanos de município do Nordeste do Brasil. Cien Saude Colet 2013;18:1109-18.

28. Ramaswamy V, Desarbo WS, Reibstein DJ, et al. An empirical pooling approach for estimating marketing mix elasticities with PIMS data. Marketing Sci 1993;12:103-24.

29. Muthén LK, Muthén BO. Mplus user's guide. 7th edn. Los Angeles, CA: Muthén \& Muthén, 1998-2012.

30. Sanne B, Torp S, Mykletun A, et al. The Swedish Demand-ControlSupport Questionnaire (DCSQ): factor structure, item analyses, and internal consistency in a large population. Scand J Public Health 2005;33:166-74.

31. Kasl SV. The influence of the work environment on cardiovascular health: a historical, conceptual, and methodological perspective. $J$ Occup Health Psychol 1996:1:42-56.

32. Söderfeldt B, Söderfeldt M, Muntaner C, et al. Psychosocial work environment in human service organizations: a conceptual analysis and development of the demand-control model. Soc Sci Med 1996;42:1217-26.

33. Gyorkos C, Becker J, Massoudi K, et al. The impact of personality and culture on the job demands-control of job stress. Swiss $J$ Psychol 2012;71:21-8.

34. Karasek R, Theorell T. Healthy work: stress, productivity, and the reconstruction of working life. New York, NY: Basic Books, 1990.

35. Jonge J, Dollard MF, Dormann C, et al. The Demand Control Model: specific demands,specific control, and well-defined groups. Int $J$ Stress Manag 2000;7:269-87.

36. Levi L, Bartley M, Marmot M, et al. Stressors at the workplace: theoretical models. Occup Med 2000;15:69-106.

37. Phipps DL, Malley C, Ashcroft DM, Denham LP, Characteristics J. Job characteristics and safety climate: the role of effort-reward and demand-control-support models. J Occup Health Psychol 2012;17:279-89.

38. Bakker AB, van Veldhoven M, Xanthopoulou D. Beyond the DemandControl model: thriving on high job demands and resources. $J$ Personnel Psychol 2010;9:3-16.

39. Seligman ME, Maier SF. Failure to escape traumatic shock. J Exp Psychol 1967;74:1-9. 